\title{
Papers
}

\section{Olfactory detection of human bladder cancer by dogs: proof of principle study}

Carolyn M Willis, Susannah M Church, Claire M Guest, W Andrew Cook, Noel McCarthy, Anthea J Bransbury, Martin R T Church, John C T Church

\begin{abstract}
Objective To determine whether dogs can be trained to identify people with bladder cancer on the basis of urine odour more successfully than would be expected by chance alone. Design Experimental, "proof of principle" study in which six dogs were trained to discriminate between urine from patients with bladder cancer and urine from diseased and healthy controls and then evaluated in tests requiring the selection of one bladder cancer urine sample from six controls.

Participants 36 male and female patients (age range 48-90 years) presenting with new or recurrent transitional cell carcinoma of the bladder (27 samples used for training; 9 used for formal testing); 108 male and female controls (diseased and healthy, age range 18-85 years -54 samples used in training; 54 used for testing).

Main outcome measure Mean proportion of successes per dog achieved during evaluation, compared with an expected value of 1 in 7 (14\%).

Results Taken as a group, the dogs correctly selected urine from patients with bladder cancer on 22 out of 54 occasions. This gave a mean success rate of $41 \%$ (95\% confidence intervals $23 \%$ to $58 \%$ under assumptions of normality, $26 \%$ to $52 \%$ using bootstrap methods), compared with $14 \%$ expected by chance alone. Multivariate analysis suggested that the dogs' capacity to recognise a characteristic bladder cancer odour was independent of other chemical aspects of the urine detectable by urinalysis.

Conclusions Dogs can be trained to distinguish patients with bladder cancer on the basis of urine odour more successfully than would be expected by chance alone. This suggests that tumour related volatile compounds are present in urine, imparting a characteristic odour signature distinct from those associated with secondary effects of the tumour, such as bleeding, inflammation, and infection.
\end{abstract}

\section{Introduction}

The hypothesis that dogs may be able to detect malignant tumours on the basis of odour was first put forward by Williams and Pembroke in a letter to the Lancet in 1989. ${ }^{1}$ Their thinking arose from a consultation with a woman who claimed to have sought medical help as a direct result of her dog's inordinate interest in a skin lesion, which subsequently proved to be a malignant melanoma. Since then similar anecdotal claims of detection of skin cancer, and of malignancies of internal organs such as breast and lung, have appeared in the press and in a further letter to the Lancet. $^{2-4}$
Although these anecdotal events remain unsupported by experimental evidence, the concept that dogs can "smell" cancer is not unreasonable. Tumours produce volatile organic compounds, which are released into the atmosphere through, for example, breath and sweat..$^{5-9}$ Some of these volatile organic compounds are likely to have distinctive odours; even when present in minute quantities, they could be detectable by dogs, with their exceptional olfactory acuity. ${ }^{10-13}$

Interest in the exploitation of volatile organic compounds for diagnostic purposes is growing, ${ }^{5}{ }^{6}$ suggesting that dogs have the potential to make a contribution in the field of oncology, providing that a scientific basis to the anecdotal reports can be established. With this in mind, we designed a study to determine whether dogs can detect cancer by olfactory means. We chose human bladder cancer as the experimental model ${ }^{14}$ on the basis that tumour related volatile organic compounds are released into urine, ${ }^{8}$ which can be readily collected and presented to dogs for training and testing purposes. Our aim was to train dogs to recognise an odour, or combination of odours (an "odour signature"), characteristic of bladder cancer but distinct from those associated with the secondary effects of the tumour, such as bleeding, inflammation, infection, and necrosis. These factors are present in a multitude of non-malignant conditions of the urinary tract and elsewhere in the body and must be ignored by the dogs if discrimination is to be attained. We assessed the dogs' abilities to detect bladder cancer, once trained, by comparison of their success rate with that expected by chance alone, in choosing one cancer urine placed randomly among six controls in blinded experiments.

We should emphasise that our objective at this stage was to conduct a simple, yet stringent, "proof of principle" study to answer the question, "Can dogs be trained to detect bladder cancer more successfully than would be expected by chance alone?" This was not an attempt to assess or predict the clinical usefulness of this hypothesised capability of dogs.

\section{Methods}

\section{Training of the dogs}

Six dogs of varying breeds and ages completed a seven month period of training. All were familiar with obedience commands, but none had been previously trained for search or scent discrimination tasks. We made no attempt to include dogs with a particular suitability for scent discrimination.

The training objective was to enable the dogs to discriminate between urine from patients with bladder cancer and urine from diseased and healthy people, using samples entirely new to them, 
so as to preclude simple memory recognition of participants' unique odour signatures. Dogs were trained to detect ("alert to" or "indicate") one urine sample from a patient with bladder cancer placed among six control specimens. We selected this task format (of being able to select one urine from seven) with reference to data on dogs' behaviour..$^{15}$ Training was by operant conditioning, using the clicker training method $^{15}$; the dogs were taught to indicate the appropriate sample by lying beside it. Early recognition of the tumour scent was achieved by using search and find games, which were gradually replaced by discrimination phases of increasing complexity. Urine from patients with bladder cancer was presented sequentially against water, diluted urine from healthy people, undiluted urine from healthy controls, urine (containing blood) from menstruating women, and urine from patients with non-malignant active or recent urological disease or other disease. Samples were not pooled at any stage. Two of the dogs were located 150 miles from the study centre and were trained and tested with dried urine samples. The remaining four Buckinghamshire based dogs were provided with freshly defrosted, liquid specimens throughout.

\section{Participant selection}

We recruited patients from hospitals within the Buckinghamshire Hospitals NHS Trust and additional healthy controls from among staff and their families. All participants gave written, informed consent. Thirty six patients (23 men, age range 48-90, mean age $69 ; 13$ women, age range 49-90, mean age 74) presenting with new or recurrent transitional cell carcinoma of the bladder gave urine before surgical intervention. We used 27 of these samples in training and the remaining nine for evaluation (table 1).

A total of 108 diseased and healthy control participants supplied urine (54 men, age range 18-85, mean age $45 ; 54$ women, age range $18-85$, mean age 40 ); we used 54 samples in training and 54 during evaluation (table 1). We required people aged over 30 to have had recent cystoscopy to exclude visible bladder malignancy. We included male controls aged over 50 only if recent prostate histology had been negative for cancer. We excluded patients with premalignant urological disease or a history of urological carcinoma. A history of other malignancy was acceptable providing the patient was now considered diseasefree. All other past or current medical conditions were permissible. We made no exclusions on the basis of drugs, menstrual cycle, ethnicity, diet, alcohol consumption, smoking habits, exposure to chemicals, or findings on urinalysis. However, we recorded details of all of these factors for each participant, in case we needed to consider their influence on the composition and odour of the urine at any stage.

\section{Analysis and processing of urine samples}

After urinalysis (Multistix 10 SG, Bayer Corporation, NY, USA), we refrigerated fresh urine specimens within 45 minutes and froze them 2-32 hours later as $0.5 \mathrm{ml}$ aliquots in glass vials. We then stored them at $-40^{\circ} \mathrm{C}$ for up to five months. For presentation to the dogs, samples were defrosted and pipetted on to filter paper in Petri dishes (58 x $15 \mathrm{~mm}$ ) and used either immediately in a wet state or within four weeks after overnight air drying and storage at room temperature.

\section{Evaluation of trained dogs}

\section{Test samples}

We assessed the dogs for their ability to select correctly one urine sample from a bladder cancer patient placed among six control samples (the same task as used in their training); all samples were new to the dogs. For statistical reasons, we used nine test panels, each with one positive sample and six controls, to test each dog (table 1). In selecting the samples for each panel, we first sex matched the controls to the cancer sample to circumvent hormonal influences. We also age matched ( \pm 8 years) at least one control with the cancer sample. Most panels also had a second age matched control ( \pm 12 years). All age matched controls had some form of urological disease. Most panels included a further two control samples from people with urological problems.

The choice of which control we assigned to each bladder cancer sample was then further determined by the results of urinalysis. Where possible, we tried to match the quantity of blood present in at least one of the controls to that of the cancer urine. We were not able to provide matches for all other abnormalities present in the cancer urine specimens.

\section{Conduct of tests}

One investigator, working in a building separate from the dog testing area, prepared the test samples, labelling Petri dishes for each run with the letters A to $\mathrm{G}$, from a randomly ordered list. Different investigators then placed the Petri dishes under single use, ventilated plastic pots, in positions (a minimum of $50 \mathrm{~cm}$ apart) along a floor grid numbered 1 to 7 , by using a second random number list. Random lists were produced by NMcC, who was not present during testing, using Stata software. The trainer allowed the dog to smell the samples until he or she was satisfied by its indication and then noted the position of the selected urine. Fresh samples and new random orders were used for each test run and for each dog. Disposable vinyl gloves were used throughout and changed each time a new urine sample was handled in order to prevent cross-contamination. We recorded all test runs on videotape.

\section{Statistical analysis}

The primary outcome measure was the mean proportion of successes for each dog, compared with an expected value of 1 in 7 (approximately 0.143). Given the small dataset and the uncertainty of the form of the data, we estimated $95 \%$ confidence intervals by using both normal assumptions and bootstrap techniques. The bootstrap intervals reported were bias corrected and accelerated bootstrap confidence intervals, ${ }^{16}$ as implemented in Stata, and based on 19999 replications.

The study had power in excess of $95 \%$ to show a statistically significant result $(\mathrm{P}<0.05)$ for a mean success rate of at least $55 \%$, irrespective of the method of analysis used. We assessed power by 1000 stochastic simulations of the experiment with each dog having an expected success rate of between $45 \%$ and $60 \%$ (mean 55\%). We analysed results by $t$ test and bootstrap techniques, to ensure that the power was adequate under both forms of analysis.

We applied a conditional logistic regression model to assess whether factors measured on urinalysis (presence of blood, leucocytes, protein, ketones, bilirubin, nitrites, or urobilinogen) might confound the association between participants' cancer status and selection of their urine by the dogs. Being selected by the dogs was the outcome of this analysis, with cancer status and urinalysis findings as explanatory variables. We simplified the full model by backward stepwise removal of variables not significant at $\mathrm{P}<0.1$ and then compared the association between cancer and selection in the final multivariate model with the univariate model, including only cancer status. We used a $t$ test and rank sum test to assess the effect of the method of training (two dogs trained on dried urine samples compared with four dogs trained on wet urine). 
Table 1 Baseline characteristics of participants selected for blinded test runs

\begin{tabular}{|c|c|c|c|}
\hline Sample & Relevant current medical history and pathology & Age (years) & Urinalysis abnormalities \\
\hline \multicolumn{4}{|c|}{ Run 1 (female) } \\
\hline TCC 1 & Bladder TCC: G3 pT2 with flat CIS* & 50 & Bld (3+), Leu (3+), Pro, Bil, Ket, Nit, Uro \\
\hline $\mathrm{c} 1$ & Interstitial cystitis & 55 & Bld $(1+)$, Leu $(3+)$ \\
\hline$\overline{\mathrm{C} 2}$ & Recurrent UTIS, dyspareunia & 47 & Bld $(1+)$ \\
\hline $\mathrm{c} 3$ & Macroscopic haematuria & 35 & Leu (trace) \\
\hline $\mathrm{C} 4$ & Cysto-uterine fistula & 24 & Bld $(3+)$ \\
\hline C 5 & Aseptic microscopic haematuria & 25 & Bld (NHT), Leu (trace), Ket \\
\hline$\overline{\mathrm{C} 6}$ & Healthy control & 22 & Bld (NHT) \\
\hline \multicolumn{4}{|l|}{ Run 2 (male) } \\
\hline TCC 2 & Bladder TCC: G3 $\mathrm{pT}_{\mathrm{T}} \mathrm{b}^{*}$, ureteric renal stone & 48 & Bld $(3+)$, Leu (trace), Pro, Ket \\
\hline $\mathrm{c} 7$ & Prostatism, microscopic haematuria (PSA 0.6) & 49 & Bld $(2+)$ \\
\hline$\overline{C 8}$ & Urinary retention, post-coital haematuria (PSA 1.5) & 43 & None \\
\hline$\overline{c 9}$ & Dysuria, haematuria, scrotal pain & 39 & None \\
\hline C 10 & Kidney stone & 35 & None \\
\hline C 11 & Healthy control & 28 & None \\
\hline C 12 & Healthy control & 22 & None \\
\hline \multicolumn{4}{|c|}{ Run 3 (female) } \\
\hline TCC 3 & Bladder TCC: G2 $\mathrm{pTa}^{*}$ & 59 & Bld $(3+)$ \\
\hline C 13 & Microscopic haematuria & 58 & Bld (HT) \\
\hline C 14 & Multiple sclerosis, suprapubic catheter & 55 & Bld (NHT), Leu (3+), Nit \\
\hline $\bar{c} 15$ & Suprapubic pain, microscopic haematuria, red trigone & 50 & Bld (NHT), Pro \\
\hline$\overline{c 16}$ & Scalp psoriasis & 27 & Bld $(3+)$ \\
\hline $\mathrm{c} 17$ & Healthy control, menstruating & 26 & Bld $(1+)$ \\
\hline C 18 & Healthy control, menstruating & 25 & None \\
\hline \multicolumn{4}{|c|}{ Run 4 (female) } \\
\hline $\operatorname{TCC} 4$ & Bladder TCC: G2 $\mathrm{pTa}^{*}$ & 68 & Bld $(3+)$, Leu $(1+)$, Pro \\
\hline $\mathrm{C} 19$ & Type 2 diabetes, renal colic, macroscopic haematuria & 66 & None \\
\hline C 20 & Interstitial cystitis & 56 & Bld $(2+)$ \\
\hline c 21 & Stress incontinence, recurrent UTIS & 32 & Bld (NHT) \\
\hline$\overline{c 22}$ & Urinary frequency, menstruating & 26 & None \\
\hline $\mathrm{C23}$ & Healthy control, menstruating & 24 & None \\
\hline $\bar{c} 24$ & Healthy control & 23 & None \\
\hline \multicolumn{4}{|c|}{ Run 5 (male) } \\
\hline TCC 5 & Bladder TCC: G2 $\mathrm{pTa}^{*}$ & 70 & Bld (HT) \\
\hline$\overline{c 25}$ & Kidney stone, prostatism (PSA 2), BPH & 63 & Bld $(2+)$ \\
\hline c 26 & Ureteric renal stone, stent removed 2 months before sample & 43 & None \\
\hline C 27 & Macroscopic haematuria (Crohn's disease) & 37 & None \\
\hline $\bar{c} 28$ & UTI symptoms, hydronephrotic duplex kidney & 28 & None \\
\hline $\bar{c} 29$ & Healthy control & 26 & None \\
\hline $\mathrm{C} 30$ & Healthy control & 25 & None \\
\hline \multicolumn{4}{|c|}{ Run 6 (male) } \\
\hline TCC 6 & Bladder TCC: G2 pT1 $\mathrm{b}^{*}$ & 49 & Bld (3+), Leu (trace), Pro, Bil, Ket \\
\hline $\mathrm{C} 31$ & Urethral stricture (type 2 diabetes) & 50 & None \\
\hline$c 32$ & Ureteric renal stone, stent & 45 & Bld (3+), Leu (trace), Pro, Bil \\
\hline $\mathrm{c} 33$ & Ureteric renal stone removed 2 months before sample & 34 & Bld (3+), Pro, Nit \\
\hline c 34 & Post-coital macroscopic haematuria, meatal stenosis & 27 & Pro \\
\hline c 35 & Healthy control & 25 & None \\
\hline$c 36$ & Healthy control & 22 & None \\
\hline \multicolumn{4}{|c|}{ Run 7 (female) } \\
\hline TCC 7 & Bladder TCC: G3 pT2* & 82 & Bld $(3+)$, Leu (2+), Pro, Nit \\
\hline $\mathrm{C} 37$ & Urinary incontinence & 76 & Bld (HT) \\
\hline$c 38$ & Urethral stricture & 71 & Bld $(2+)$, Leu $(3+)$, Nit \\
\hline $\mathrm{c} 39$ & Recurrent UTIs, microscopic haematuria & 62 & Bld $(2+)$ \\
\hline $\mathrm{C} 40$ & Urinary urgency and frequency, cystoscopy 1 week before sample & 46 & Bld (HT), Leu $(3+)$ \\
\hline C 41 & Healthy control, menstruating & 27 & None \\
\hline $\mathrm{C} 42$ & Healthy control, menstruating & 26 & Bld (1+) \\
\hline \multicolumn{4}{|c|}{ Run 8 (male) } \\
\hline TCC 8 & Bladder TCC: G3 pT2* & 76 & Bld (NHT) \\
\hline C 43 & $\mathrm{BPH}$, indwelling catheter & 68 & Bld $(3+)$, Leu $(1+)$, Nit \\
\hline C 44 & Prostatism, BPH & 58 & None \\
\hline $\mathrm{C} 45$ & Microscopic haematuria & 37 & Bld (3+), Pro \\
\hline$\overline{c 46}$ & Healthy control & 29 & None \\
\hline$c 47$ & Healthy control & 24 & None \\
\hline $\mathrm{C} 48$ & Penile warts & 24 & Ket \\
\hline \multicolumn{4}{|c|}{ Run 9 (female) } \\
\hline TCC 9 & Bladder TCC: G3 $\mathrm{pTa}^{*}$ & 85 & Bld $(3+)$ \\
\hline C 49 & Recurrent UTIs, microscopic haematuria & 86 & Bld $(1+)$, Ket \\
\hline C 50 & Recurrent UTIS, ureteric renal stone & 73 & Bld (HT), Leu (trace), Ket \\
\hline $\mathrm{c} 51$ & Microscopic haematuria and dysuria & 68 & Bld (HT), Leu (trace) \\
\hline$\overline{c 52}$ & Stress incontinence & 41 & Bld (HT) \\
\hline$\overline{c 53}$ & Healthy control, menstruating & 26 & Bld $(3+)$ \\
\hline$c 54$ & Healthy control & 26 & Bld (NHT) \\
\hline
\end{tabular}

Bil=bilirubin; Bld=blood; BPH=benign prostatic hyperplasia; C=control; HT=haemolysed trace; Ket=ketone; Leu=leucocytes; NHT=non-haemolysed trace; Nit=nitrite; Pro=protein; PSA=prostate specific antigen; TCC=transitional cell carcinoma; Uro=urobilinogen; UTI=urinary tract infection. ${ }^{*}$ For details of grading and staging of TCCs, see reference 14. 


\section{Results}

Table 2 gives the results for the formal test runs. Taken as a single group, the dogs correctly selected the positive bladder cancer urine on 22 of 54 occasions. This gave a mean success rate of $41 \%$ (95\% confidence intervals $23 \%$ to $58 \%$ under assumptions of normality and $26 \%$ to $52 \%$ using bootstrap methods), compared with $14 \%$ expected by chance.

The association between presence of cancer and selection by the dogs was slightly stronger in the multivariate model, which also included presence of blood and ketones, than in the univariate model. This indicated that the association was not due to confounding with factors measured on urinalysis.

The four dogs trained on wet urine specimens ( $50 \%$ correct) seemed to perform better than the two dogs trained on dried samples $(22 \%$ correct; $\mathrm{P}=0.03$ by $t$ test, $\mathrm{P}=0.06$ by rank sum test). However, the small numbers involved limit confidence in the certainty of this observation.

\section{Discussion}

\section{Summary of findings}

Given the extraordinary claims made about dogs detecting cancer on the basis of odour, ${ }^{1-4}$ our aim was to design and conduct a simple, yet stringent, experiment to establish whether dogs have this capability. We achieved the successful detection of urine samples from patients with bladder cancer $41 \%$ of the time (rather than the $14 \%$ expected by chance alone), providing convincing evidence that dogs do, indeed, have this ability. Multivariate analysis suggests that the dogs' capacity to recognise an odour signature characteristic of bladder cancer is independent of other chemical aspects of the urine detectable by urinalysis, such as the presence of blood. Although this multivariate model does not fully allow for the lack of independence in the data, because each dog did the same set of tests, it is, nevertheless, able to assess possible confounding of a specific cancer signature with other features of bladder cancer urine. Exactly what the chemical composition of the cancer odour signature is we can only speculate at present. Evidence from gas chromatography and mass spectroscopy studies indicates that elevated levels of formaldehyde, alkanes, and benzene derivatives occur with some cancers, ${ }^{5-8}$ but other volatile molecules are probably produced as well.

\section{Rationale for training approach}

When we embarked on this project we had no relevant peer reviewed publications to refer to. The trainers on the team were experienced at teaching dogs to scent-match, but this was not the task being demanded of the dogs here. We needed them to learn to recognise an odour signature for cancer from among the hundreds present in urine, without recourse to the "pure" source of the odour. This makes it very different from training dogs to detect, for example, drugs or explosives. At the beginning of the study we considered using surplus tumour material obtained during surgery. We dismissed this, however, largely because the tissue could not be chemically fixed without irrevocably altering the smell, and the use of unfixed tissue had serious health and safety implications for the dog trainers.

Having decided that we would concentrate on urine as the source of tumour derived volatile organic compounds, we then had to consider whether to use each participant's urine sample separately or whether to pool those of the cancer patients and, separately, those of the controls. Although pooling might have led to a greater concentration of the desired odour signature, we foresaw some important disadvantages and pitfalls. Firstly, we had no idea whether certain foods, drinks, or drugs, for example, may obscure, interfere with, or even mimic, the odour of tumour related compounds. Only by taking detailed histories from each participant, and introducing each sample separately, could we gradually eliminate these possibilities. Secondly, pooling specimens would lead to many fewer samples being available for the dogs to smell. The very real possibility then existed that dogs would merely scent-match with known samples, rather than learn to pick out the distinctive odour signature common to the cancer urines. Lastly, and perhaps most importantly, we were concerned that "rogue" control specimens from people with undiagnosed cancer elsewhere in the body may be inadvertently added to pooled samples. We did, in fact, have an occasion during training in which all dogs unequivocally indicated as positive a sample from a participant recruited as a control on the basis of negative cystoscopy and ultrasonography. The consultant responsible for the patient was sufficiently concerned to bring forward further tests, and a transitional cell carcinoma of the right kidney was discovered.

We next had to consider the physical state of the urine when presented to the dog. We felt that air dried samples would have greater applicability in a clinical setting, by virtue of easy handling, transport, and storage. However, the overnight drying process may result in the loss of volatile organic compounds important to the overall odour signature. We therefore opted to train one cohort of dogs on wet samples and another on dried samples. When tested, the dogs trained on liquid urine performed significantly better, suggesting that the more volatile molecules are of importance in the cancer odour signature.

Table 2 Urine samples selected by the six dogs during evaluation

\begin{tabular}{|c|c|c|c|c|c|c|c|}
\hline \multirow[b]{2}{*}{ Run } & \multirow{2}{*}{$\begin{array}{c}\text { Mongrel } \\
\text { (age 6, M)* }\end{array}$} & \multirow{2}{*}{$\begin{array}{c}\text { Labrador } \\
\text { (age 7, F)* }\end{array}$} & \multicolumn{3}{|c|}{ Working strain Cocker Spaniel† } & \multirow[b]{2}{*}{ Papillon (age 7, F)† } & \multirow[b]{2}{*}{ Correct } \\
\hline & & & (age 1.5, M) & (age 2, F) & (age 5, F) & & \\
\hline 1 & TCC 1 & TCC 1 & TCC 1 & TCC 1 & TCC 1 & TCC 1 & 6 \\
\hline 2 & C 11 & TCC 2 & C 11 & TCC 2 & TCC 2 & TCC 2 & 4 \\
\hline 3 & C 14 & C 13 & C 17 & C 16 & C 15 & C 13 & 0 \\
\hline 4 & C 23 & C 23 & TCC 4 & TCC 4 & C 22 & TCC 4 & 3 \\
\hline 5 & C 28 & TCC 5 & TCC 5 & TCC 5 & TCC 5 & TCC 5 & 5 \\
\hline 6 & C 34 & C 31 & C 31 & TCC 6 & TCC 6 & C 31 & 2 \\
\hline 7 & C 41 & C 41 & TCC 7 & C 42 & C 42 & C 38 & 1 \\
\hline 8 & C 46 & C 48 & TCC 8 & C 48 & C 48 & C 47 & 1 \\
\hline 9 & C 53 & C 50 & C 54 & C 50 & C 50 & C 54 & 0 \\
\hline Correct & 1 & 3 & 5 & 5 & 4 & 4 & 22 \\
\hline
\end{tabular}




\section{What is already known on this topic}

Canine olfactory detection of cancer has been anecdotally reported but has not, until now, been the subject of scientific scrutiny

\section{What this study adds}

Dogs can be trained to distinguish patients with bladder cancer on the basis of urine odour more successfully than would be expected by chance alone

This study provides a benchmark against which future studies can be compared

However, the small sample sizes, together with other potentially confounding variables between the two groups of dogs, such as breed, age, and environmental conditions during testing, limit confidence in this observation. Further work to determine the optimum physical state for the urine will therefore be needed.

Lastly, we gave careful consideration to the selection of patients and controls. During training, we exposed the dogs to urine from patients presenting with a broad range of transitional cell carcinomas, in terms of grade and stage, as we felt this would increase their likelihood of recognising the common factor or factors. We took particular care to train the dogs with control samples containing elements likely to be present in urine from patients with bladder cancer but also commonly occurring in other non-malignant pathologies. In this way, we could teach the dogs to ignore non-cancer specific odours. This led to the inclusion of urine samples, during both training and evaluation, from a variety of patients, such as people with diabetes to control for glucose, those with chronic cystitis to deal with the influence of leucocytes and protein, and healthy menstruating women to control for blood. Given the prevalence of benign prostatic hyperplasia in the age group of men most likely to have bladder cancer, we also included this condition in both training and testing.

\section{Conclusion}

Our approach to training was vindicated by the results achieved when the dogs were formally evaluated. Despite the fact that we had not used dogs with proved scenting abilities, and despite the inclusion of age matched diseased controls, we achieved a statistically significant success rate. We learnt a great deal during the study, and we are confident that improvements in the success rate can be achieved by modifications to the training regimen. In particular, we need to work on suitable reward mechanisms when the trainers are blinded to the samples, so as not to confuse the dogs. Also, for this approach to cancer detection to have more clinical relevance, we would need to teach the dogs to respond to more than one positive sample at a time, and to have a signal for "no positive sample present."

In summary, our study provides the first piece of experimental evidence to show that dogs can detect cancer by olfactory means more successfully than would be expected by chance alone. The results we achieved should provide a benchmark against which future studies can be compared, and we hope that our approach to training may assist others engaged in similar work.

We thank all the participants who helped us with the study, Sandra Stevenson and Jan Smith for their expert training of four of the dogs, and Lezlie
Britton for expert laboratory assistance. We also thank the following people for their advice and support: the consultants who contributed patients to the study, particularly Amar Bdesha; senior nurses Glenys Newton and Hilary Baker; consultant pathologist David Bailey; and Mike Scott, Pete Smith, and Alistair Stevenson. We also acknowledge the enthusiastic support of the Trustees of the Erasmus Wilson Dermatological Research Fund.

Contributors: All authors participated in conception and design of the study, interpretation of data, and critical revision of the manuscript. SMC, JCTC, MRTC, and CMW did the patient recruitment and sample collection, and CMW was also responsible for the storage and management of the urine samples. WAC and CMG had overall responsibility for the training of the dogs. With the exception of NMcC and AJB, all authors contributed to the acquisition of data. NMcC did the statistical analysis. CMW drafted the manuscript, with assistance from SMC and NMcC. CMW is the guarantor and accepts full responsibility for the conduct of the study, had access to the data, and controlled the decision to publish.

Funding: The Department of Dermatology, Amersham Hospital, received financial support from the Erasmus Wilson Dermatological Research Fund (registered charity No 313305), which had no active role in the design or conduct of the study. The dog trainers, all employees of Hearing Dogs for Deaf People (registered charity No 293358), trained the dogs in their own time; their expenses were met through a private donation given by Derek Wilton, who did not participate in the study in any way. SMC, MRTC, and JCTC were funded by their small family company COBiRD Ltd (company No 03426189), which also contributed to the project expenses.

Competing interests: None declared.

Ethical approval: South Buckinghamshire local research ethics committee approved the study.

1 Williams H, Pembroke A. Sniffer dogs in the melanoma clinic? Lancet 1989;1:734.

2 Fraser L. Scientists put sniffer dogs on the scent of men with cancer. Sunday Telegraph 2002 June 2.

3 Dobson R. Dogs can sniff out first signs of men's cancer. Sunday Times 2003 Apr 27:5.

4 Church J, Williams H. Another sniffer dog for the clinic? Lancet 2001;358:930.

4 Church J, Williams H. Another sniffer dog for the clinic? Lancet 2001;358:930.
5 Phillips M, Gleeson K, Hughes JM, Greenberg J, Cataneo RN, Baker L, et al. Volatile organic compounds in breath as markers of lung cancer: a cross-sectional study. Lancet 1999;353:2897-8.

6 Di Natale C, Macagnano A, Martinelli E, Paolesse R, D'Arcangelo G, Roscioni C, et al. Lung cancer identification by the analysis of breath by means of an array of non-selective gas sensors. Biosens Bioelectron 2003;18:1209-18.

7 Phillips M, Cataneo RN, Ditkoff BA, Fisher P, Greenberg J, Gunawardena R, et al. Volatile markers of breast cancer in the breath. Breast J 2003;9:184-91.

8 Spanel P, Smith D, Holland TA, Al Singary W, Elder JB. Analysis of formaldehyde in the headspace of urine from bladder and prostate cancer patients using selected ion flow tube mass spectrometry. Rapid Commun Mass Spectrom 1999;13:1354-9.

9 Yamada K, Walsh N, Hara H, Jimbow K, Chen H, Ito S. Measurement of eumelanin precursors metabolites in the urine as a new marker for melanoma metastases. Arch Dermatol 1992;128:491-4.

10 Schoon GAA, De Bruin JC. The ability of dogs to recognise and cross-match human odours. Forensic Sci Int 1994;69:111-8.

11 Schoon GAA. A first assessment of the reliability of an improved scent identification line-up. J Forensic Sci 1998;43:70-5.

12 Komar D. The use of cadaver dogs in locating scattered, scavenged human remains: preliminary field test results. J Forensic Sci 1999;44:405-8.

13 Lorenzo N, Wan T-L, Harper RJ, Hsu Y-L, Chow M, Rose S, et al. Laboratory and field experiments used to identify Canis lupus var. familiaris active odor signature chemicals from drugs, explosives, and humans. Anal Bioanal Chem 2003;376:1212-24.

14 Pashos CL, Botteman MF, Laskin BL, Redaelle A. Bladder cancer: epidemiology, diagnosis and management. Cancer Pract 2002;10:311-22.

15 Mills DS. Learning, training and behavioural modification techniques. In: Horwitz DF, Mills DS, Heath S, eds. BSAVA manual of canine and feline behavioural medicine. Gloucester: British Small Animal Veterinary Association, 2002.

16 Carpenter JR, Bithell JF. Bootstrap confidence intervals: when? which? what?-A practical guide for medical statisticians. Stat Med 2000;19:1141-64.

(Accepted 19July 2004)

bmj.com 2004;329:712

Department of Dermatology, Amersham Hospital, Amersham HP7 0JD

Carolyn M Willis senior research scientist

Susannah M Church honorary research fellow

Anthea J Bransbury associate specialist

Martin R T Church honorary research fellow

John C T Church honorary consultant

Hearing Dogs for Deaf People, Saunderton, Princes Risborough HP27 9NS

W Andrew Cook deputy chief executive

Claire M Guest operations director

Centre for Statistics in Medicine, Institute of Health Sciences, Oxford OX3 7LF

Noel McCarthy medical statistician

Correspondence to: C M Willis carolyn.willis@sbucks.nhs.uk 


\section{Commentary: Teaching dogs new tricks}

TJ Cole

\section{"You can't teach an old dogma new tricks"}

Dorothy Parker

Dogs are widely recognised as smelling smells that humans miss. Yet the idea of turning this canine skill to clinical diagnosis is novel. The study by Willis et al takes a first cautious step in testing such an idea by training dogs to detect bladder cancer from urine samples and then seeing if their detection rate when tested blind is better than expected by chance. ${ }^{1}$

The design of the trial was simple and elegant. Six dogs were trained to recognise urine samples from patients with bladder cancer compared with diseased and healthy sex matched controls. Each dog was then offered a set of seven urine samples, from a person with cancer and six controls, and they identified the sample they considered to be different by lying next to it. This process was repeated eight times, so each dog effectively rolled a seven sided die nine times and by chance ought to have been successful one seventh of the time (14\%). In practice the success rate was almost three times higher, 22 out of 54 or $41 \%$. This is a highly significant result, especially with a fancy bootstrap confidence interval.

The study was carefully designed to include several features to minimise bias, and it is hard to fault the study in this respect. On balance the results are unambiguous-dogs can be trained to recognise and flag an unusual smell in the urine of patients with bladder cancer. This gives the lie to Dorothy Parker's epigram.

Some intriguing findings for dog lovers are in the detail. The dogs were deliberately chosen to cover a range of breeds and ages, and they had no particular skills in scent discrimination. The papillon performed almost as well as the three cocker spaniels, while the mongrel did worst. The two dogs trained with dried urine samples fared less well (four successes out of 18) than the others who were trained with intact samples (18 out of 36).

Looking at the results by patient again showed some striking differences. Patient 1 was correctly identified by all six dogs, whereas patients 3 and 9 were consistently missed. This may be a fatigue effect, as the results were worse in later tests (exact $\mathrm{P}$ for trend $=0.0006$ ), or it may simply indicate that the strength of the urine signal varies from one patient to another.

The most intriguing finding was the control patient seen during the training phase, whose urine sample was consistently identified by the dogs as a case. Despite the fact that the patient had negative cystoscopy and ultrasonography results, the consultant was sufficiently impressed by the dogs' performance to test the patient again and found a kidney carcinoma.

Competing interest: TJC owns a chocolate labrador.

1 Willis CM, Church SM, Guest CM, Cook WA, McCarthy N, Bransbury AJ, et al. Olfactory detection of human bladder cancer by dogs: a proof of principle study. BMJ 2004;329:712-4.

Centre for Paediatric Epidemiology and Biostatistics, Institute of Child Health, London WC1N 1EH

$\mathrm{T} \mathrm{J}$ Cole professor of medical statistics

tim.cole@ich.ucl.ac.uk 